\title{
A Biopsychosocial Approach to Examine Mexican American Adolescents' Academic Achievement and Substance Use
}

\author{
YANG QU, ADRIANA GALVÁN, ANDREW J. FULIGNI, \\ AND EVA H. TELZER
}

Taking a comprehensive biopsychosocial approach and using a two-wave longitudinal design, this study examines the relation between brain development and the social environment in Mexican American youth's $(N=41.56$ percent female) academic achievement and substance use. We find that both Mexican American youth's structural brain development and social environment uniquely contribute to their adjustment. Specially, smaller hippocampal volume and parental cultural socialization each uniquely predict better academic achievement. Moreover, smaller nucleus accumbens volume and less affiliation with deviant peers each uniquely predict less substance use. These findings underscore the independent contributions of biological and psychosocial factors in youth's adjustment. The study provides a new biopsychosocial perspective on Mexican American youth's well-being.

Keywords: academic achievement, cultural socialization, brain structure, substance use

Mexican Americans are the largest and fastest growing ethnic minority group in the United States, making up about 17 percent (fifty-six million people) of the U.S. population. Challenges associated with immigration, discrimination, and lower socioeconomic status place Mexican American youth at particularly high risk for poor adjustment, including school dropout and substance use. For example, Mexican American youth's school dropout rates are approximately double that of any other ethnic group (U.S. Department of Commerce 2006). Such disadvantage in $\mathrm{K}-12$ schools results in negative long-term problems in school trajectories, such that Mexican American students have the lowest postsecondary enrollment rate (24 percent). In addition, relative to adolescents in other ethnic groups, Mexican American adolescents have higher rates of substance use, begin using drugs at an earlier age, and show greater risk for developing drug use disorders in adulthood due to early drug use on-

Yang Qu is a postdoctoral scholar at Stanford University. Adriana Galván is associate professor of psychology and faculty member of the Brain Research Institute at University of California, Los Angeles. Andrew J. Fuligni is professor in the Department of Psychology and faculty member of the Brain Research Institute and the Semel Institute for Neuroscience and Human Behavior at the University of California, Los Angeles. Eva H. Telzer is assistant professor in the Department of Psychology and Neuroscience at the University of North Carolina, Chapel Hill.

(C) 2018 Russell Sage Foundation. Qu, Yang, Adriana Galván, Andrew J. Fuligni, and Eva H. Telzer. 2018. “A Biopsychosocial Approach to Examine Mexican American Adolescents' Academic Achievement and Substance Use." RSF: The Russell Sage Foundation Journal of the Social Sciences 4(4): 84-97. DOI: 10.7758/RSF.2018.4.4.05. Direct correspondence to: Yang Qu at yangqu@stanford.edu, 450 Serra Mall, Stanford, CA 94305; Adriana Galván at agalvan@psych.ucla.edu; Andrew J. Fuligni at afuligni@ucla.edu; and Eva H. Telzer at ehtelzer@unc. edu, 235 E. Cameron Ave., Chapel Hill, NC 27599.

Open Access Policy: RSF: The Russell Sage Foundation Journal of the Social Sciences is an open access journal. This article is published under a Creative Commons Attribution-NonCommercial-NoDerivs 3.0 Unported License. 
set (Eaton et al. 2006; Johnston et al. 2009; Marsiglia et al. 2005). It is therefore important to identify protective factors that are associated with better academic achievement and less substance use.

\section{A BIOPSYCHOSOCIAL APPROACH TO STUDY MEXICAN AMERICAN ADOLESCENTS}

To address ethnic disparities in academic achievement and substance use, it is critical to systematically examine biological and psychosocial factors that influence Mexican American adolescents. Past research has taken either a biological or a psychosocial approach to understand adolescents' well-being, highlighting the importance of both biological (for example, brain structure) and psychosocial (for example, social environment) factors in adolescents' adjustment. For example, advances in neuroimaging techniques allow researchers to examine how social relationships get "under the skin" (Fuligni and Telzer 2013). In this endeavor, countless exciting findings have revealed how neural structure and function are related to adolescent adjustment. However, it is also acknowledged that examining brain structure and function alone cannot inform us how social environments are related to the neurobiology of the developing child.

Although both biological and psychosocial approaches provide valuable insights to our understanding of minority adolescents' wellbeing, few studies to date combine these two approaches to provide a more comprehensive perspective on adolescent development. In the absence of systematic investigation, it remains unclear whether biological and psychosocial factors play a unique role in minority adolescents' adjustment. This study therefore took an integrative biopsychosocial approach to systematically examine how biological (youth's brain development) and psychosocial (parents' cultural socialization and deviant peer association) factors are uniquely related to Mexican American youth's academic achievement and substance use. Findings will provide valuable insights into promoting Mexican American children's well-being during adolescence, an important period of brain development and socialization.
Brain Structure and Adolescents' Well-being Neuroimaging research has demonstrated dramatic brain development during adolescence. Prior research has characterized functional brain development in Mexican American adolescents, with attention to the role of family and peer contexts (Telzer et al. 2013a, 2013b; Telzer et al. 2015; Qu et al. 2015). However, no work to date has examined Mexican American adolescents' structural brain development and the potential unique effects of structural changes and social environment on adolescents' adjustment. This is a limitation because neural changes during adolescence not only involve changes in brain function, but also changes in brain structure. Although functional and structural changes often go hand in hand, they also uniquely predict adjustment outcomes. Thus, individual differences in brain structure may also predict individual differences in academic and psychological adjustment.

An interesting phenomenon during adolescence is the parallel between loss of cortical gray matter and improvement in cognitive abilities. Although the whole brain may reach its maximum size around the age of five years, grey and white matter subcomponents continue to undergo significant changes throughout adolescence (Giedd et al. 1999; Sowell et al. 2003; Gogtay et al. 2004). Specifically, cortical gray matter volume begins to decline in late childhood or early adolescence, and white matter shows a linear increase over the same period. For example, in a large-scale longitudinal neuroimaging study, a curvilinear change in grey matter was found, such that it increased from childhood to adolescence, and then decreased in adolescence and into adulthood (Giedd et al. 1999). The decline in gray matter is thought to be driven by synaptic pruning, a process through which unused synapses are eliminated to increase the efficiency of neuronal transmissions (Huttenlocher 1990). Therefore, lower gray matter volume may indicate greater pruning and more mature neural development.

A key neural region related to learning and memory is the hippocampus, a brain region in the medial temporal lobe (Cohen and Eichenbaum 1993; Maguire, Frackowiak, and Frith 1997; Maguire et al. 2000). Empirical studies 
have examined the association between hippocampal volume and adolescents' adjustment, which seem to yield inconsistent findings at first glance. Although some studies suggest that larger hippocampal volume is linked to better memory and learning (Erickson et al. 2011), others find the opposite pattern (Foster et al. 1999). A key factor overlooked in previous studies is the developmental stage. Indeed, a metaanalysis across development found age-related changes in such association (Van Petten 2004). Although hippocampal volume and memory have a weak positive relationship among adults (see Golomb et al. 1994; Raz et al. 1998), a negative relationship between hippocampal volume and memory was significant for studies with children and adolescents (see Riggins et al. 2012; Sowell et al. 2001). Similarly, this significant negative association between hippocampal volume and memory performance has been found in healthy young adults (Chantôme et al. 1999; Foster et al. 1999; Pruessner et al. 2007). Such association is thought to be explained by the degree of neural pruning that occurs during childhood and adolescence, with smaller gray matter volume indicating more pruning (that is, neural specialization). Thus, smaller hippocampus volume may indicate greater brain maturation and is related to educational advantages.

The nucleus accumbens plays a central role in reward seeking, risk taking, substance use, and addictive behaviors (Casey, Getz, and Galván 2008; Galván 2010; Knutson et al. 2001). Previous functional MRI studies have examined the association between nucleus accumbens activation and adolescents' adjustment, suggesting that greater activity in the nucleus accumbens is related to greater risk taking (for example, Galván et al. 2007; Qu et al. 2015). Only a few studies have used structural MRI to investigate the link between nucleus accumbens volume and risk taking. Accumulating evidence reveals a preliminary positive relationship between the two. For example, young adults who use cannabis showed larger nucleus accumbens volume than non-drug users (Gilman et al. 2014). Moreover, nucleus accumbens volume is positively associated with frequency of drinking among adolescents (Thayer et al. 2012). Interestingly, the developmental decline in reward sensitivity from late adolescence to young adulthood is accompanied by a decrease in nucleus accumbens volumes (Urošević et al. 2012). Thus, smaller nucleus accumbens volume may be associated with less reward-seeking behaviors such as substance use.

\section{SOCIAL ENVIRONMENT AND}

ADOLESCENTS' WELL-BEING

In a separate body of work, researchers have taken a psychosocial approach to identify factors in social environment that play a role in Mexican American adolescents' well-being. Based on findings from this line of research, parents and peers serve as two key socialization agents. Drawing on this literature, this study focuses on two important factors that may influence adolescents' academic achievement and substance use-parents' cultural socialization and adolescents' association with deviant peers.

In ethnic minority families, one socialization goal for parents is to help their children develop a strong connection to their ethnic heritage and understanding of cultural values (Hughes et al. 2006; Parke and Buriel 2006). Therefore, parents engage in related practices. Specifically, parents talk to their children about their country of origin, celebrate cultural holidays and historical events, and expose children to culturally relevant books, arts, and music (Hughes and Chen 1997; Knight et al. 1993). Because these practices are embedded in daily parent-child interactions, parents' cultural socialization is also a protective factor for minority adolescent wellbeing. Indeed, empirical studies suggest that parental cultural socialization practices are related to adolescents' development of ethnic pride and identification (Rivas-Drake, Hughes, and Way 2009), and ultimately lead to better academic and behavioral outcomes, such as more school engagement and less antisocial behavior (Hughes et al. 2009).

In addition, as children enter adolescence, they spend more time with their peers (Larson and Verma 1999). Their academic and psychological adjustment is thus also influenced by their peer groups. For example, exposure to delinquent peers may lead to increased involvement in substance abuse due to the processes of imitation, social learning, and peer pressure (Deater-Deckard 2001; Dishion, Patterson, and Griesler 1994; Moffitt 1993). Indeed, deviant 
peer association is one of the strongest predictors of substance use in adolescence (Barrera et al. 2002; Fergusson, Swain-Campbell, and Horwood 2002; Jenkins 1996). Importantly, among Mexican American adolescents, strong family values relate to less substance use because adolescents are less likely to associate with deviant peers (Telzer, Gonzales, and Fuligni 2014). Avoidance of deviant peers is thus an important protective factor in adolescents' substance use.

\section{CURRENT STUDY}

Building on prior literature, the current research took an integrative biopsychosocial approach to systematically examine the role of biological (youth's brain development) and psychosocial (parents' cultural socialization and deviant peer association) factors on Mexican American youth's adjustment, focusing on their academic achievement and substance use. Given substantial variation among Mexican American adolescents, this study investigated how individual differences in structural brain development and social environment were predictive of individual differences in academic and psychological adjustment, rather than comparing Mexican American adolescents with their counterparts in other ethnic groups. Findings not only will provide insights into how biological and psychosocial factors are related to Mexican American youth's adjustment, but also have the potential to be generalized to other minority groups.

Our first goal was to examine the role of brain structure and social environment in Mexican American adolescents' academic achievement. Given that effective pruning leads to greater reduction in gray matter volume, we hypothesized that smaller volume in the hippocampus, a key region related to memory and learning, would predict better academic achievement. In addition, based on research on minority adolescents (Hughes et al. 2006), we hypothesized that parents' cultural socialization would contribute to youth's better academic achievement.

Our second goal was to investigate the mediating role through which brain structure and social environment play a role in Mexican American adolescents' academic achievement.
Specifically, we focused on the adolescents' positive work habits. We hypothesized that smaller hippocampal volume and parents' cultural socialization would facilitate better work habits among adolescents, which ultimately promotes better academic achievement.

Our third goal was to examine the role of brain structure and social environment in Mexican American adolescents' substance use. We focused on the nucleus accumbens, a region consistently related to reward seeking and risk taking. Based on prior research, we predicted that smaller volume in the nucleus accumbens would be related to less substance use (Thayer et al. 2012). Given that deviant peer association consistently predicts adolescents' substance use across different studies, we further hypothesized that Mexican American adolescents' association with more deviant peers would be related to more severe substance use (Barrera et al. 2002; Telzer, Gonzales, and Fuligni 2014).

\section{METHODS}

Forty-one Mexican American adolescents ( mean age at $\mathrm{T} 1=15.24$ years, range $=14.02$ to 16.25 years, $\mathrm{SD}=0.54,56$ percent girls) participated in a two-wave longitudinal study. Most participants were from low-SES families with the majority of fathers ( 87 percent) and mothers (78 percent) receiving a high school diploma or less. At T1, adolescents reported on their parents' cultural socialization practices and their affiliation with deviant peers. To measure their brain structure, adolescents underwent a structural magnetic resonance imaging (sMRI) scan one year later (T2). Adolescents reported on their substance use at $\mathrm{T} 2$, and we obtained adolescents' grade point average (GPA) from school records and teachers report of adolescents' work habits. Participants completed written consent and assent in accordance with the Institutional Review Board.

\section{Measures}

Youth reported on their parents' cultural socialization practices using the ethnic-racial socialization scale at T1 (Hughes and Chen 1997). This scale is a self-report scale designed to measure the amount of cultural socialization the adolescent has received from parents in the last year, and has been used in studies on Mexican 
American parents' cultural socialization (for example, Hughes 2003). Using four items, adolescents reported how frequently $(1=$ never to $5=$ six or more times) their parents engaged in cultural socialization in the past year (for example, "In the past year, how many times have your parents encouraged you to read books concerning the history or traditions of your ethnicity?"). Their responses were averaged, higher scores indicating greater cultural socialization $(\alpha=0.74)$.

For deviant peer association, at T1, youth indicated the number of their friends who engage in risky activities using a measure previously used among Mexican American youth (Barrera et al. 2002). This measure included fifteen deviant behaviors, such as got drunk or high, cheated on school tests, started a fight with someone, and stole something. For each behavior, adolescents reported on how many of their friends engaged in this risky activity in the last month on a five-point scale ( $1=$ "none", $5=$ "almost all"). Their responses were averaged, with higher scores indicating more deviant peers $(\alpha=0.91)$.

At the end of T2, teachers reported on adolescents' work habits based on criteria for marks for Los Angeles Unified School District. Work habits in four subjects, including math, English, science, and social science, were collected. Work habits capture a wide range of adolescent school behavior, such as effort, responsibility, and attendance. For each subject, students received an E (excellent; for example, "Makes explicit effort to examine work using both teacher-generated and self-generated criteria."), S (satisfactory; for example, "Makes effort to examine work using teacher-generated criteria."), or U (unsatisfactory; for example, "Makes use only of teacher-generated criteria to examine work on an inconsistent basis."), which was then converted to numbers ( $\mathrm{E}=2, \mathrm{~S}$ $=1$, and $U=0$ ). For each participant, work habits across four subjects were averaged, with higher scores indicating better work habits.

For adolescents' academic achievement, at the end of T2, adolescents' GPA was obtained from school records. Grades were originally in letters and converted to a four-point scale $(0=$ F to $4=\mathrm{A}$ ).

At T2, adolescents reported on their use of substances on the Center for Disease Control and Prevention Youth Risk Behavior Survey Questionnaire, a common measure that has been shown to be valid and reliable for Mexican American youth (Kerr et al. 2003). This in-depth form asks about youth's lifetime use (for example, if you have ever tried marijuana, how old were you when you tried it for the first time?) for the following substances: cigarettes, alcohol (including beer, wine, wine coolers, and liquor that does not include sips of wine for religious purposes), marijuana (for example, pot, weed, grass, hash), cocaine (for example, powder, crack, or freebase), crystal meth (also called ice or glass), and other illegal drugs (for example, LSD, PCP, ecstasy, mushrooms, speed, or heroin). To examine substance use, an index was created that indicates the type of substance the adolescent had ever tried lifetime, where $0=$ never tried any type of substance, 1 = tried legal substances (alcohol or cigarettes) at least once, 2 = tried marijuana at least once, and $3=$ tried hard substances (cocaine, crystal meth, or other illegal drugs) at least once. Higher scores indicate more severe substance use.

Demographic information on adolescents' gender and parents' educational attainment were collected at T1. The primary caregiver indicated the highest educational attainment for each parent, which was assessed using a tenpoint scale $(1=$ "some elementary school", $10=$ "graduated from medical, law, or graduate school"). A composite score that averages father's and mother's highest educational attainment was calculated to represent parents' average educational attainment, with higher scores indicating higher educational attainment. Both adolescents' gender and parents' educational attainment were taken into account in all analyses.

\section{Structural MRI Data Acquisition}

Imaging data were collected using a 3.0 Tesla Siemens Trio MRI scanner. High resolution T1weighted brain images were acquired using a 3D magnetization-prepared rapid-acquisition gradient echo (MPRAGE) scan with 160 contiguous axial slices, collected in ascending fashion parallel to the anterior and posterior commissures, echo time $(\mathrm{TE})=2.1 \mathrm{~ms}$, repetition time $(\mathrm{TR})=2300 \mathrm{~ms}$, field of view $(\mathrm{FOV})=256$ 
$\mathrm{mm}$, acquisition matrix $192 \mathrm{~mm} \times 192 \mathrm{~mm}$, sagittal plane, and slice thickness $=1 \mathrm{~mm}$.

\section{Segmentation and Volumetric Analysis}

Segmentation and volumetric analysis of the hippocampus and nucleus accumbens were performed using FMRIB's (Oxford Center for Functional MRI of the Brain) Integrated Registration and Segmentation Tool (FIRST) in FMRIB's Software Library (FSL) version 4.1.9 (Patenaude et al. 2007a, 2007b). FIRST is a semiautomated, model-based subcortical tool using a Bayesian framework.

First, for each participant's MPRAGE, this method ran a two-stage affine registration to a standard space template (Montreal Neurological Institute space) with one millimeter resolution using twelve degrees of freedom and a subcortical mask to exclude voxels outside the subcortical regions. Second, the left and right hippocampus and nucleus accumbens were segmented with thirty, forty, and fifty modes of variation, respectively. To achieve accurate segmentation, the FIRST methodology models 317 manually segmented and labeled T1 brain images from normal children, adults, and pathological populations as a point distribution model with the geometry and variation of the shape of each structure submitted as priors. Volumetric labels are parameterized by a 3D deformation of a surface model based on multivariate Gaussian assumptions. FIRST searches through linear combinations of shape modes of variation for the most probable shape (that is, brain structure) given the intensity distribution in the T1-weighted image, and specific brain regions are extracted (for further description of the method, see Patenaude et al. 2007a, $2007 \mathrm{~b}$ ). Modes of variation are optimized based on leave-one-out cross-validation on the training set, and they increase the robustness and reliability of the results (Patenaude et al. 2007b). The segmentations were visually checked for errors. Finally, boundary correction was run, a process that classifies boundary voxels as belonging to the structure or not based on a statistical probability (z-score $>3.00 ; p<.001$ ).

The volume of each participant's brain region was measured in millimeters cubed. Volumes were estimated separately for the left and right hemispheres. The left and right volumes for the hippocampus and nucleus accumbens were examined in the current analyses.

\section{RESULTS}

Our analyses examined how brain structure and social environment relate to adolescents' academic achievement and substance use.

\section{Descriptive Statistics of Academic \\ Achievement and Substance Use}

We first examined youth's academic achievement. The average GPA was moderately low $(M=2.20$; that is, C- average), with substantial variability within the group $(S D=1.03$, range $=$ .19 to 3.75$)$. On average, girls tended to perform better in school $(M=2.45)$ compared with boys $(M=1.88), t(39)=1.78, p=.08$. Parents' educational attainment was not related to youth's academic achievement, $r=.06, p=.72$.

Next, we investigated youth's substance use. The frequency for lifetime substance use is presented in table 1 . Nearly two-thirds of the sample engaged in substance use in their lifetime, the majority in marijuana. Males and females did not differ in their substance use, $t(39)=.50$, $p=.62$. Moreover, substance use did not vary across parents' educational attainment, $r=-.22$, $p=.16$.

Bivariate correlations between all study variables are presented in table 2. Hippocampal and nucleus accumbens volumes were not correlated to each other. Whereas hippocampal volume was correlated with work habits and GPA but not substance use, nucleus accumbens volume was associated with substance use but not GPA or work habits. Parents' cultural so-

Table 1. Current Stage of Substance Use in Mexican American Youth

\begin{tabular}{lccr}
\hline Stage & Male & Female & Total (\%) \\
\hline 0 & 9 & 6 & $15(36.6)$ \\
1 & 1 & 6 & $7(17.1)$ \\
2 & 4 & 8 & $12(29.3)$ \\
3 & 4 & 3 & $7(17.1)$ \\
\hline
\end{tabular}

Source: Authors' calculations.

Note: $0=$ no substance use, $1=$ substances that are legal for adults (such as tobacco and alcohol), $2=$ marijuana, and $3=$ other illicit substances (such as cocaine, crystal meth, heroin, and speed). 
Table 2. Bivariate Correlations

\begin{tabular}{|c|c|c|c|c|c|c|c|c|c|}
\hline & 1 & 2 & 3 & 4 & 5 & 6 & 7 & 8 & 9 \\
\hline 1. Left hippocampal volume & - & & & & & & & & \\
\hline $\begin{array}{l}\text { 2. Right hippocampal } \\
\text { volume }\end{array}$ & $.51^{* *}$ & - & & & & & & & \\
\hline $\begin{array}{l}\text { 3. Left nucleus accumbens } \\
\text { volume }\end{array}$ & .06 & .24 & - & & & & & & \\
\hline $\begin{array}{l}\text { 4. Right nucleus accumbens } \\
\text { volume }\end{array}$ & .04 & .19 & $.57^{* * *}$ & - & & & & & \\
\hline 5. Cultural socialization & -.24 & .11 & -.10 & -.04 & - & & & & \\
\hline 6. Deviant peer association & .02 & .17 & .16 & .07 & $.37^{*}$ & - & & & \\
\hline 7. Work habits & $-.41^{* *}$ & -.18 & -.08 & -.16 & $.33^{*}$ & .02 & - & & \\
\hline 8. Academic achievement & $-.44^{* *}$ & -.10 & -.09 & -.11 & $.41^{* *}$ & .07 & $.96^{* * *}$ & - & \\
\hline 9. Substance use & .01 & .26 & $.31^{*}$ & .20 & .22 & $.50 * *$ & -.16 & -.10 & - \\
\hline
\end{tabular}

Source: Authors' calculations.

${ }^{* * *} p<.001,{ }^{* *} p<.01,{ }^{*} p<.05$

Figure 1. Left Hippocampal Volume and Youth's Academic Achievement

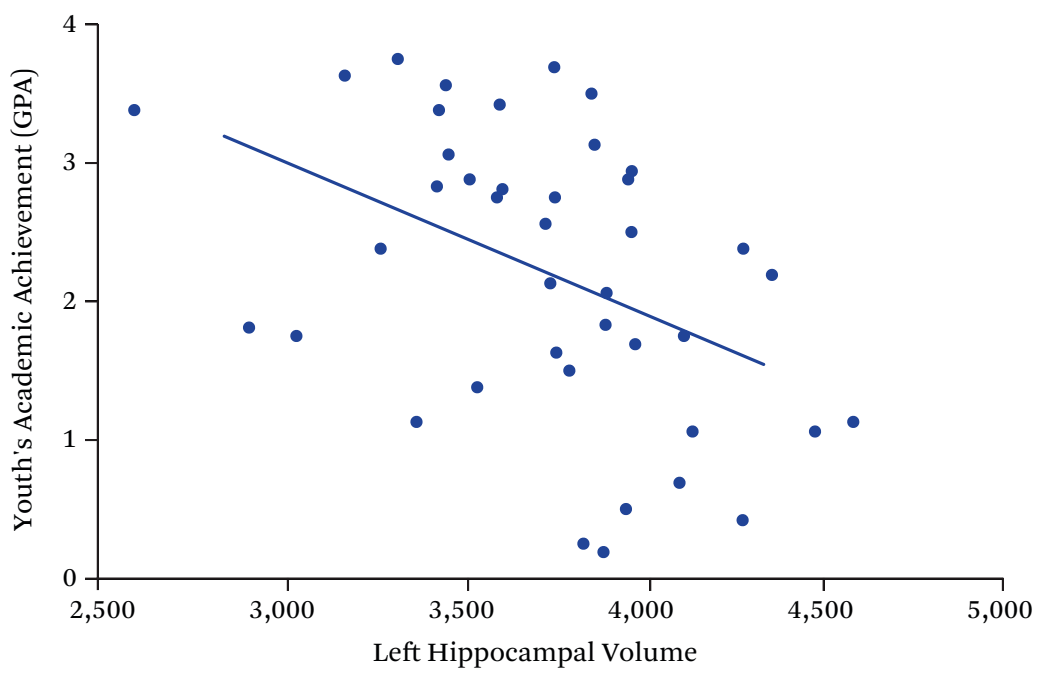

Source: Authors' calculations.

cialization and adolescents' deviant peer association were not related to hippocampal and nucleus accumbens volumes.

\section{The Role of Brain Structure and Social}

Environment in Academic Achievement

Our first analysis examined the role of biological and psychosocial factors in Mexican American youth's academic achievement. To this end, we conducted regression analyses with youth's brain structure, parents' cultural socialization, and deviant peer association predicting youth's GPA. Specifically, we focused on volume in the hippocampus, a region related to memory and learning. Consistent with previous research, our results indicated that smaller volume in the left hippocampus was associated with better academic achievement (that is, higher GPA), $p<.01$ (figure 1).

When parents' cultural socialization and adolescents' association with deviant peers were included in the regression model, results indicated that parents' cultural socialization was positively associated with youth's academic 
Table 3. Regression Analysis for Academic Achievement

\begin{tabular}{lcccc}
\hline Predictor & $B$ & $S E(B)$ & $\beta$ & $t$ \\
\hline Gender & .09 & .16 & .09 & .55 \\
Parents' education & -.03 & .08 & -.05 & -.34 \\
Left hippocampal volume & -.001 & .00 & -.43 & $-2.83^{* *}$ \\
Parents' cultural socialization & .50 & .17 & .44 & $2.92^{* *}$ \\
Deviant peer association & -.15 & .22 & -.10 & -.67 \\
\hline
\end{tabular}

Source: Authors' calculations.

Note: For youth's gender, $-1=$ male and $1=$ female.

${ }^{* *} p<.01$

Figure 2. Hippocampal Volume, Work Habits, and Academic Achievement

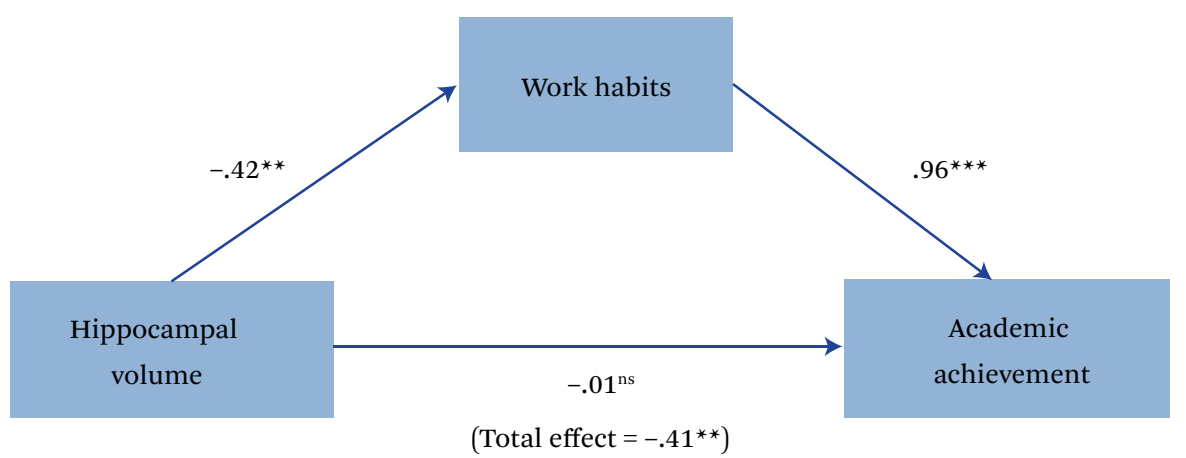

Source: Authors' calculations.

${ }^{* *} p<.01 ;{ }^{* *} p<.001 ; \mathrm{ns}=$ not significant

achievement (table 3). Importantly, both hippocampal volume and parents' cultural socialization had unique effects on youth's academic achievement. Affiliation with deviant peers was not related to academic achievement.

To understand how hippocampal volume and parents' cultural socialization are related to youth's academic achievement, we examined positive work habits. To test whether work habits mediate the link between hippocampal volume and academic achievement as well as that between parents' cultural socialization and academic achievement, we conducted two mediation analyses using bias-corrected bootstrapping resampling techniques (Preacher and Hayes 2008).

In the first set of mediation analyses, the independent variable was hippocampal volume, the dependent variable was youth's academic achievement, and the mediator was their work habits. Based on five thousand bootstrap resamples, the indirect path from hippocampal volume to academic achievement via work habits was significant: indirect effect $=-.40,95$ percent CI: $(-.74,-.11)$ (figure 2). The link between hippocampal volume and academic achievement was no longer significant after work habits were taken into account, which showed a 97 percent reduction in the total effect.

In the second set of analyses, the independent variable was parents' cultural socialization, the dependent variable was youth's academic achievement, and the mediator was their work habits. Based on five thousand bootstrap resamples, the indirect path from parents' cultural socialization to work habits to academic achievement was significant: indirect effect = .28 , 95 percent CI: $(.04, .54)$ (figure 3$)$. The reduction in the total effect between cultural socialization and academic achievement was 72 percent, which remained significant after taking into account work habits. 
Figure 3. Parents' Cultural Socialization, Work Habits, and Academic Achievement

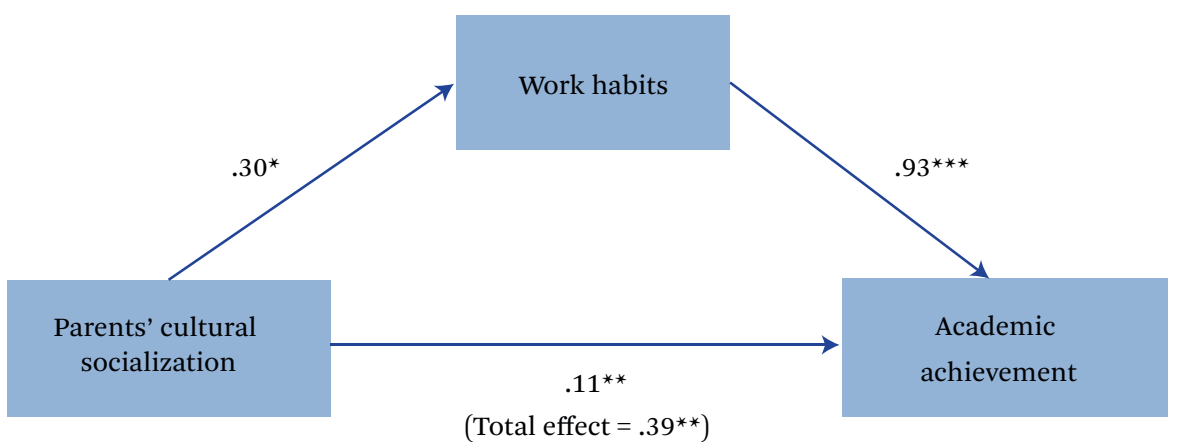

Source: Authors' calculations.

${ }^{*} p<.05 ;{ }^{* *} p<.01 ;{ }^{* * *} p<.001$

Table 4. Regression Analysis for Substance Use

\begin{tabular}{lcccc}
\hline Predictor & $B$ & $S E(B)$ & $\beta$ & $t$ \\
\hline Gender & .01 & .16 & .01 & .03 \\
Parents' education & -.09 & .09 & -.14 & -.95 \\
Left nucleus accumbens volume & .003 & .002 & .33 & $2.23^{*}$ \\
Parents' cultural socialization & -.02 & .19 & -.02 & -.13 \\
Deviant peer association & .68 & .25 & .44 & $2.76^{* *}$ \\
\hline
\end{tabular}

Source: Authors' calculations.

Note: For youth's gender, $-1=$ male and $1=$ female.

${ }^{*} p<.05 ;{ }^{* *} p<.01$

\section{The Role of Brain Structure and Social}

\section{Environment in Substance Use}

Next, we examined the role of biological and psychosocial factors in Mexican American youth's substance use. Given prior research, we focused on the nucleus accumbens, a region involved in reward seeking and risk taking. Similar to analyses on academic achievement, we conducted regression analyses with youth's nucleus accumbens volume, parents' cultural socialization, and deviant peer association predicting youth's substance use. As shown in table 4, consistent with our hypotheses, smaller volume in the nucleus accumbens was associated with less substance use among Mexican American youth.

When parents' cultural socialization and deviant peer association were included in the regression model, results indicated that more deviant peers was positively associated with youth's substance use (table 4). Importantly, both nucleus accumbens volume and deviant peers had unique effects on Mexican American youth's substance use. Parents' cultural socialization was not related to substance use.

\section{DISCUSSION}

With an increasing population, Mexican American adolescents' disadvantage in school and heightened substance use have drawn attention from researchers, educators, and policymakers. In this study, focusing on variation within a Mexican American sample, we took a biopsychosocial approach to examine how brain development and social environment are uniquely associated with adolescents' academic achievement and substance use. Adolescents who showed smaller hippocampal volume and whose parents provided greater cultural socialization showed better academic achievement. Moreover, smaller nucleus accumbens volume and less affiliation with deviant peers are related to less substance use. Taken together, our findings provide empirical 
evidence to demonstrate that both Mexican American youth's brain development and their social environment are uniquely associated with their academic achievement and substance use.

\section{The Role of Brain Structure and Social Environment in Academic Achievement}

Smaller hippocampal volume was associated with better academic achievement. Despite evidence suggesting a negative correlation between hippocampal volume and memory during adolescence (Van Petten 2004), prior neuroimaging research has not examined the link between hippocampal volume and adolescents' actual performance in school, making it unclear whether hippocampal volume plays a role in academic achievement. We found a significant negative association between hippocampal volume and adolescents' GPA. Our findings thus underscore the important role of the hippocampus in adolescents' actual school performance. Consistent with synaptic pruning during adolescence, smaller hippocampal volume may indicate more effective pruning and greater brain maturation, which is linked to adolescents' better academic adjustment.

Previous studies have suggested the important role of parents' cultural socialization in promoting minority adolescents' performance in school. For example, greater cultural socialization is related to greater school engagement among minority adolescents (for example, Hughes et al. 2009). In line with these studies, adolescents who reported their parents providing more cultural socialization at T1 showed higher GPA at T2. Although it is possible that these parents provide more general support and guidance to their adolescents, research suggests that parents' cultural socialization, a unique parenting practice in ethnic minority families, may play a distinctive role in minority adolescents' adjustment over and above other family factors (for example, parental warmth) (Hughes et al. 2006). Parents' cultural socialization predicted adolescents' academic achievement above and beyond the effect of hippocampal volume. This finding contributes to the rich literature revealing that parents' transmission of cultural values in daily life benefits adolescents' academic adjustment, highlighting the unique role of parents' cultural socialization in shaping adolescents' learning. Such cultural transmission may be particularly important during adolescence, a time when adolescents actively seek to pursue their ethnic identity (for example, French et al. 2006). Interventions designed at promoting Mexican American adolescents' school performance can focus on encouraging parents to convey cultural values and heritages to their children.

The link between hippocampal volume and academic achievement and the link between parents' cultural socialization and academic achievement was mediated by adolescents' work habits. Specifically, adolescents who showed smaller hippocampal volume and who reported greater cultural socialization exhibited better work habits, as reported by their teachers. Moreover, better work habits were associated with higher GPA. In contrast, adolescents who showed larger hippocampal volume or who reported less cultural socialization exhibited worse work habits, which was associated with lower GPA. These findings suggest that smaller hippocampal volume and heightened parents' cultural socialization may facilitate adolescents' self-regulation in school. It is also possible that adolescents' self-regulation in school, such as their work habits, play a role in decreasing hippocampal volume. Moreover, these findings are in line with prior studies showing that greater self-regulation is related to better academic achievement (for a review, see Zimmerman 1990). In this study, adolescents' academic achievement and teacher-report work habits were highly correlated, highlighting that teachers largely incorporate judgments of work habits when assigning grades. Although our mediation analyses suggest that the link between hippocampal volume and academic achievement and the link between parents' cultural socialization and academic achievement might be due to better work habits, future studies also need more precise measurement of these habits and examine other mechanisms underlying these associations.

\section{The Role of Brain Structure and Social Environment in Substance Use}

We also examined the role of brain development and social environment in Mexican Amer- 
ican adolescents' substance use. Neuroimaging studies have paid considerable attention to how nucleus accumbens activation is related to adolescents' psychological adjustment. For example, previous fMRI research suggests that greater nucleus accumbens activation in the context of risk taking is related to adolescents' greater risk taking and substance use in real life (Galván et al. 2007). Structural MRI research has found that smaller nucleus accumbens volume, which may indicate less reward sensitivity, is associated with less cannabis (Gilman et al. 2014) and alcohol use (Thayer et al. 2012). Moreover, longitudinal declines in nucleus accumbens volume are related to declines in selfreported reward sensitivity from adolescence to young adulthood (Urošević et al. 2012). Consistent with these studies, we find that smaller nucleus accumbens volume predicts less substance use. Our finding, together with those from prior studies, suggest that smaller nucleus accumbens volume is related to adolescents' reward sensitivity and substance use.

Adolescents' social environment also played an important role in their substance use. Prior studies suggest that adolescents' risk taking may be largely influenced by their peer groups (Barrera et al. 2002; Fergusson, Swain-Campbell, and Horwood. 2002; Jenkins 1996). For example, minority adolescents whose peers use illegal drugs are more likely to do the same (Brook et al. 1998). Consistent with this line of research, we find that adolescents who have more deviant peers use more illicit drugs. This finding suggests that the characteristics of peer groups uniquely influence adolescents' risktaking behavior and highlights the detrimental role of deviant peer association in adolescents' adjustment. Given emerging evidence that peers modulate neural activation in the rewardrelated regions (for example, Chein et al. 2011; Telzer et al. 2015), it is possible that adolescents' association with deviant peers may play a role in brain structure.

\section{LIMITATIONS AND FUTURE STUDIES}

This study has several limitations, pointing to directions for future studies. First, given the small sample size, future studies are needed to examine this neurodevelopmental process in a larger sample of adolescents. Although forty- one participants is an acceptable sample size in neurobiological research, the number is considered relatively small in psychosocial research, which needs larger sample sizes to detect the association between psychosocial factors and adolescents' outcomes. However, our findings on the role of psychosocial factors in adolescents' adjustment are consistent with well-documented results based on survey studies with large samples (Barrera et al. 2002; Fergusson, Swain-Campbell, and Horwood 2002; Hughes et al. 2009; Jenkins 1996). Second, this study focuses on within-group variations among Mexican American adolescents, not across ethnic groups. These findings, then, may not necessarily be generalized to other ethnic groups. Moreover, although we took a biopsychosocial approach and examined adolescents' brain structure, parents' cultural socialization, and deviant peer association, we did not include other biological or psychosocial factors that may also influence Mexican American adolescents' academic achievement and substance use. For example, in earlier reports of the same sample, we examined the association between nucleus accumbens activation and adolescents' risk taking both concurrently and longitudinally (Telzer et al. 2013a, 2013b; Qu et al. 2015). Together, this study and our prior work suggest that structural brain development, functional brain development, and peer and family contexts play a key role in Mexican American adolescents' adjustment. Other psychosocial factors, such as parents' academic expectation, parental substance use, and adolescents' ethnic identity, may also play a role in adolescents' academic achievement and substance use. Therefore, future studies are needed to capture more aspects of psychosocial factors to better understand the causes of adolescents' problem behavior.

\section{CONCLUSIONS}

Taken together, the current study builds on a significant body of literature highlighting the importance of biological and psychosocial factors in adolescents' well-being. Our findings provide a new contribution to the growing literature and suggest that Mexican American youth's brain development and their social environment are uniquely associated with their 
academic achievement and substance use. It is important to highlight that our findings are based on a multi-informant, multimethod, and multidimensional design. We used adolescents' self-reports (that is, cultural socialization and deviant peer association), along with neuroimaging assessment of their brain structure, to predict teacher reports of work habits and actual performance in school. Multiple dimensions of adolescents' adjustment, including academic achievement and substance use, were also assessed. This comprehensive design provides a new biopsychosocial perspective on understanding Mexican American youth's wellbeing, with the potential to be generalized to and have implications for other minority groups.

\section{REFERENCES}

Barrera, Manuel Jr., Hazel M. Prelow, Larry E. Dumka, Nancy A. Gonzales, George P. Knight, Marcia L. Michaels, Mark W. Roosa, and JennYun Tein. 2002. "Pathways from Family Economic Conditions to Adolescents' Distress: Supportive Parenting, Stressors Outside the Family and Deviant Peers." Journal of Community Psychology 30(2): 135-52.

Brook, Judith S., David W. Brook, Mario Dela Rosa, Luis Fernan Doduque, Edgar Rodriguez, Ivan D. Montoya, and Martin Whiteman. 1998. "Pathways to Marijuana Use Among Adolescents: Culturual/Ecological, Family, Peer, and Personality Influences." Journal of the American Academy of Child and Adolescent Psychiatry 37(7): 759-66.

Casey, B. J., Sarah Getz, and Adriana Galván. 2008. "The Adolescent Brain." Developmental Review 28(1): 62-77.

Chantôme, Martine, Pierre Perruchet, Dominique Hasboun, Didier Dormont, Mokhran Sahel, Nader Sourour, Abderrezak Zouaoui, Claude Marsault, and Michel Duyme. 1999. “Is There a Negative Correlation Between Explicit Memory and Hippocampal Volume?" Neurolmag 10(5): 589-95.

Chein, Jason M., Dustin Albert, Lia O’Brien, Kaitlyn Uckert, and Laurence Steinberg. 2011. “Peers Increase Adolescent Risk Taking by Enhancing Activity in the Brain's Reward Circuitry." Developmental Science 14(2): F1-10.

Cohen, Neal J., and Howard Eichenbaum. 1993. Memory, Amnesia, and the Hippocampal System. Cambridge, Mass.: MIT Press.
Deater-Deckard, Kirby. 2001. “Annotation: Recent Research Examining the Role of Peer Relationships in the Development of Psychopathology." Journal of Child Psychology and Psychiatry 42(5): 565-79.

Dishion, Thomas J., Gerald R. Patterson, and Pamela C. Griesler. 1994. "Peer Adaptations in the Development of Antisocial Behavior: A Confluence Model." In Aggressive Behavior: Current Perspectives, edited by L. R. Huesmann. New York: Plenum.

Eaton, Danice K., Laura Kann, Steve Kinchen, James Ross, et al. 2006. "Youth Risk Behavior Surveillance Summaries-United States, 2005." Morbidity and Mortality Weekly Report 55 (SS-5): 1-108. http://www.cdc.gov/mmwr/preview/mmwrhtml /ss5505a1.htm.

Erickson, Kirby I., Michelle W. Voss, Ruchika S. Prakash, Chandramallika Basake, et al. 2011. “Exercise Training Increases Size of Hippocampus and Improves Memory." Proceedings of the National Academy of Sciences 108(7): 3017-22.

Fergusson, David M., Nicola R. Swain-Campbell, and L. John Horwood. 2002. “Deviant Peer Affiliations, Crime and Substance Use: A Fixed Effects Regression Analysis." Journal of Abnormal Child Psychology 30(4): 419-30.

Foster, Jonathan K., Andrew Meikle, Gregory Goodson, Andrew R. Mayes, Matthew Howard, and Sandra I. Sünram, Enis Cezayirli, and Neil Roberts. 1999. "The Hippocampus and Delayed Recall, Bigger Is Not Necessarily Better?" Memory 7(5-6): 715-32.

French, Sabine E., Edward Seidman, LaRue Allen, and J. Lawrence Aber. 2006. "The Development of Ethnic Identity During Adolescence." Developmental Psychology 42(1): 1-10.

Fuligni, Andrew J., and Eva H. Telzer. 2013. “Another Way Family Can Get in the Head and under the Skin: The Neurobiology of Helping the Family." Child Development Perspectives 7(3): 138-42.

Galván, Adriana. 2010. “Adolescent Development of the Reward System." Frontiers in Human Neuroscience 4: 6. DOI: 10.3389/neuro.09.006.2010.

Galván, Adriana, Todd Hare, Henning Voss, Gary Glover, and J. B. Casey. 2007. "Risk-Taking and the Adolescent Brain: Who Is at Risk?" Developmental Science 10(2): F8-14.

Giedd, Jay N., Jonathan Blumenthal, Neal O. Jeffries, F. X. Castellanos, Hong Liu, and Alex Zijdenbos, Tomás Paus, Alan C. Evans, and Judith L. Rapo- 
port. 1999. “Brain Development During Childhood and Adolescence: A Longitudinal MRI Study." Nature Neuroscience 2(2): 861-63.

Gilman, Jodi M., John K. Kuster, Sang Lee, Myung Jung Lee, Byoung Wwoo Kim, Nikos Makris, Andre van der Kouwe, Anne J. Blood, and Hans C. Breiter. 2014. “Cannabis Use Is Quantitatively Associated with Nucleus Accumbens and Amygdala Abnormalities in Young Adult Recreational Users." Journal of Neuroscience 34(16): 5529-38.

Gogtay, Nitin, Jay N. Giedd, Leslie Lusk, Kiralee M. Hayashi, et al. 2004. “Dynamic Mapping of Human Cortical Development During Childhood Through Early Adulthood." Proceedings of the National Academy of Sciences 101(21): 8174-79.

Golomb, James, Alan Kluger, Mony J. de Leon, Steven $\mathrm{H}$. Ferris, Antonio Convit, Mary S. Mittelman, Jacob Cohen, Henry Rusinek, Susan De Santi, and Ajax E. George. 1994. "Hippocampal Formation Size in Normal Human Aging: A Correlate of Delayed Secondary Memory Performance." Learning and Memory 1(1): 45-54.

Hughes, Diane. 2003. “Correlates of African American and Latino Parents' Messages to Children About Ethnicity and Race: A Comparative Study of Racial Socialization." American Journal of Community Psychology 31(1-2): 15-33.

Hughes, Diane, and Lisa Chen. 1997. “When and What Parents Tell Children About Race: An Examination of Race-Related Socialization Among African American Families." Applied Developmental Science 1(4): 200-14.

Hughes, Diane, James Rodriguez, Emilie P. Smith, Deborah J. Johnson, Howard C. Stevenson, and Paul Spicer. 2006. "Parents' Ethnic-Racial Socialization Practices: A Review of Research and Directions for Future Study." Developmental Psychology 42(5): 747-70.

Hughes, Diane, Dawn Witherspoon, Deborah RivasDrake, and Nia West-Bey. 2009. “Received Ethnic-Racial Socialization Messages and Youths' Academic and Behavioral Outcomes: Examining the Mediating Role of Ethnic Identity and Self-Esteem." Cultural Diversity and Ethnic Minority Psychology 15(2): 112-24.

Huttenlocher, Peter R. 1990. “Morphometric Study of Human Cerebral Cortex Development." Neuropsychologia 28(6): 517-27.

Jenkins, Jeanne E. 1996. “The Influence of Peer Affiliation and Student Activities on Adolescent Drug Involvement." Adolescence 31(122): 297-306.
Johnston, Lloyd D., Patrick M. O'Malley, Jerald G. Bachman, and John E. Schulenberg. 2009. Monitoring the Future National Survey Results on Drug Use, 1975-2008, vol. 1, Secondary School Students. NIH Publication No. 09-7402. Bethesda, Md.: National Institute on Drug Abuse.

Kerr, Melissa H., Kenneth Beck, Teresa Downs Shattuck, Candace Kattar, and Diego Uriburu. 2003. "Family Involvement, Problem and Prosocial Behavior Outcomes of Latino Youth." American Journal of Health Behavior 27(Suppl. 1): S55-65.

Knight, George P., Martha E. Bernal, Camile A. Garza, Marya K. Cota, and Katheryn A. Ocampo. 1993. "Family Socialization and the Ethnic Identity of Mexican American Children." Journal of Cross-Cultural Psychology 24(1): 99-114.

Knutson, Brian, Charles M. Adams, Grace W. Fong, and Daniel Hommer. 2001. "Anticipation of Increasing Monetary Reward Selectively Recruits Nucleus Accumbens." Journal of Neuroscience 21(16): 1-5.

Larson, Reed W., and Suman Verma. 1999. "How Children and Adolescents Spend Time Across the World: Work, Play, and Developmental Opportunities." Psychological Bulletin 125(6): 70136.

Maguire, Eleanor A., Richard S. Frackowiak, and Christopher D. Frith. 1997. “Recalling Routes Around London: Activation of the Right Hippocampus in Taxi Drivers." Journal of Neuroscience 17(18): 7103-10.

Maguire, Eleanor A., David G. Gadian, Ingrid S. Johnsrude, Catriona D. Good, John Ashburner, Rrichard S. Frackowiak, and Christopher D. Frith. 2000. “Navigation-Related Structural Change in the Hippocampi of Taxi Drivers." Proceedings of the National Academy of Sciences 97(8): 4398403.

Marsiglia, Flavio F., Stephen Kulis, David A. Wagstaff, Elvira Elek, and David Dran. 2005. "Acculturation Status and Substance Use Prevention with Mexican and Mexican American Youth." Journal of Social Work Practice in the Addictions 5(1-2): 85-111.

Moffitt, Terrie E. 1993. “Adolescence-Limited and Life-Course-Persistent Antisocial Behavior: A Developmental Taxonomy." Psychological Review 100(4): 674-701.

Parke, Ross D., and Raymond Buriel. 2006. “Socialization in the Family: Ethnic and Ecological Perspectives." In Handbook of Child Psychology: So- 
cial, Emotional, and Personality Development, 6th ed., edited by Nancy Eisenberg, William Damon, and Richard M. Lerner. Hoboken, N.J.: John Wiley \& Sons

Patenaude, Brian, Stephen M. Smith, David Kennedy, and Mark Jenkinson. 2007a. "FIRSTFMRIB's Integrated Registration and Segmentation Tool." In Human Brain Mapping Conference (June): 420-28.

_. 2007b. "Bayesian Shape and Appearance Models." Technical report no. TR07BP1. Oxford: University of Oxford, FMRIB Center.

Preacher, Kristopher J., and Andrew F. Hayes. 2008. "Asymptotic and Resampling Strategies for Assessing and Comparing Indirect Effects in Multiple Mediator Models." Behavior Research Methods 40(3): 879-91.

Pruessner, Marita, J. C. Pruessner, Dirk H. Hellhammer, G. Bruce Pike, and Sonia J. Lupien. 2007. “The Associations Among Hippocampal Volume, Cortisol Reactivity, and Memory Performance in Healthy Young Men." Psychiatry Research 155(1): 1-10.

Qu, Yang, Adrdiana Galván, Andrew J. Fuligni, Matthew D. Lieberman, and Eva H. Telzer. 2015. "Longitudinal Changes in Prefrontal Cortex Activation Underlie Declines in Adolescent Risk Taking." Journal of Neuroscience 35(32): 11308-14.

Raz, Naftali, Faith M. Gunning-Dixon, Denise Head, James H. Dupuis, and James D. Acker. 1998. “Neuroanatomical Correlates of Cognitive Aging: Evidence from Structural Magnetic Resonance Imaging." Neuropsychology 12(1): 95-114.

Riggins, Tracy, Kelsey Cacic, Stacy BuckinghamHowes, Laura A. Scaletti, Betty Jo Salmeron, and Maureen M. Black. 2012. “Memory Ability and Hippocampal Volume in Adolescents with Prenatal Drug Exposure." Neurotoxicology and Teratology 34(4): 434-41.

Rivas-Drake, Deborah, Diane Hughes, and Niobe Way. 2009. "A Preliminary Analysis of Associations Among Ethnic Racial Socialization, Ethnic Discrimination, and Ethnic Identity Among Urban Sixth Graders." Journal of Research on Adolescence 19(3): 558-84.

Sowell, Elizabeth R., Dean Delis, Joan Stiles, and Terry L. Jernigan. 2001. “Improved Memory Functioning and Frontal Lobe Maturation Between Childhood and Adolescence: A Structural MRI
Study." Journal of the International Neuropsychological Society 7(3): 312-22.

Sowell, Elizabeth R., Bradley S. Peterson, Paul M. Thompson, Suzanne E. Welcome, Amy L. Henkenius, and Arthur W. Toga. 2003. "Mapping Cortical Change Across the Human Life Span." Nature Neuroscience 6(3): 309-15.

Telzer, Eva H., Andrew J. Fuligni, Matthew D. Lieberman, and Adriana Galván. 2013a. “Meaningful Family Relationships: Neurocognitive Buffers of Adolescent Risk Taking." Journal of Cognitive Neuroscience 25(3): 374-87.

. 2013b. “Ventral Striatum Activation to Prosocial Rewards Predicts Longitudinal Declines in Adolescent Risk Taking." Developmental Cognitive Neuroscience 3(1): 45-52.

—. 2015. “The Quality Of Adolescents' Peer Relationships Modulates Neural Sensitivity to Risk Taking." Social Cognitive Affective Neuroscience 10(3): 389-98.

Telzer, Eva H., Nancy Gonzales, and Andrew J. Fuligni. 2014. "Family Obligation Values and Family Assistance Behaviors: Protective and Risk Factors for Adolescent Substance Use." Journal of Youth and Adolescence 43(2): 270-83.

Thayer, Rachel E., Shirley M. Crotwell, Tiffany J. Callahan, Kent E. Hutchison, and Angela D. Bryan. 2012. "Nucleus Accumbens Volume Is Associated with Frequency of Alcohol Use Among Juvenile Justice-Involved Adolescents." Brain Sciences 2(4): 605-18.

Urošević, Snezana, Paul Collins, Ryan Muetzel, Kelvin Lim, and Monica Luciana. 2012. “Longitudinal Changes in Behavioral Approach System Sensitivity and Brain Structures Involved in Reward Processing During Adolescence." Developmental Psychology 48(5): 1488-500.

U.S. Department of Commerce. 2006. “Census Bureau, American Community Survey." Accessed November 10, 2016. http://nces.ed.gov/pubs 2008/nativetrends/tables/table_3_4a.asp.

Van Petten, Cyma. 2004. “Relationship Between Hippocampal Volume and Memory Ability in Healthy Individuals Across the Lifespan: Review and Meta-Analysis." Neuropsychologia 42(10): 1394-413.

Zimmerman, Barry J. 1990. "Self-Regulated Learning and Academic Achievement: An Overview." Educational Psychologist 25(1): 3-17. 